\title{
EDITORIAL \\ The value of health economics research in spinal cord injury
}

Spinal Cord (2013) 51, 586-587; doi:10.1038/sc.2013.47

Within all clinical areas there is an inescapable need for economic evaluation in order to determine the value of new and existing interventions, technologies and devices. This need is driven by the issue of scarcity - the fundamental analytic consideration underlying the discipline of economics - and, more specifically, limited healthcare resources. In publicly funded health-care systems, such as Australia, Canada and the United Kingdom, economic evaluation is often a mandatory requirement for technology appraisal processes, particularly regarding the reimbursement of new drugs. ${ }^{1}$

The purpose of this Editorial is not to extol the current methodologies and analytic framework of economic evaluation, or to cast them aside as dispassionate number-crunching exercises. Our intention is to highlight and discuss the key parameters (resource use, costs and health outcomes) that comprise economic evaluation. In addition, we identify current knowledge gaps that require urgent attention.

\section{RESOURCE USE AND COSTS}

A common misconception is that economic evaluation comprises an analysis of costs - and only costs. Costing is one piece of a bigger puzzle that explores the value of an intervention or technology. Determination of costing in health care is divided into three steps: (i) identification of health services resource utilization; (ii) determination of the quantity of utilization; and (iii) valuation of health services. The last step-attaching monetary value to health-care resources-cannot occur without critical information from the first two. The collection of resource utilization data provides researchers and decision makers with health system drivers, which may or may not be modifiable.

As with any condition, costing in spinal cord injury (SCI) is reliant on data about the resources utilized throughout the course of care. These resources must be identified before they can be costed, and are typically stratified into direct and indirect resources, as well as medical and non-medical resources. Resources identified and utilized must also fit within the perspective of an analysis, which may be health system, patient, insurer or society. For example, if one considers a rehabilitation system as the perspective, resources utilized could include therapists, equipment, inpatient days, as well as the management of complications. Sources for resource use data include administrative databases, clinic/medical records, literature, survey data and patient interviews. Unfortunately, most health system records do not link bedside to community. For example, patient interviews would not provide complete health system resources, whereas administrative databases would not provide data on indirect resources such as private or informal homecare. Variable sources do not allow for comprehensive data across the continuity of care for individuals with SCI. Determining robust estimates of resource use requires patient-level and prospective studies for defined cohorts, such as those conducted in other disease areas. ${ }^{2}$

In the absence of linkable data sources, clinical trials offer an option for the collection of data on health system resources utilized throughout the spectrum of SCI care. Resource utilization questions embedded in a clinical trial program provide the opportunity for high-quality collection of costing data alongside clinical data. The prospective nature of this approach (as opposed to reliance on retrospective data collection and/or secondary analysis of existing data) allows for targeted and systematic collection of appropriate resources. Such data collection methods also allow for the identification of non-health-care costs, such as lost productivity regarding paid and unpaid work, travel costs incurred when accessing health care, and the quantification of costs for informal care. ${ }^{3}$

There is a clear need for the incorporation of resource parameters into clinical trial designs; ${ }^{4}$ for an example in the context of SCI, see Sinnott et al. ${ }^{3}$ International variation in the organization and delivery of health care means that the identification and valuation of items of resource use is likely to differ, as is the determination of 'important' items of resource. Despite these differences, the need to define and value resources consumed in the treatment of SCI, and the current challenges regarding data collection, are common features throughout health-care systems.

\section{MEASURING HEALTH BENEFIT}

All economic analyses, irrespective of context, deal with inputs (resource use) and outputs (consequences/outcomes/benefits). Only when costs and outcomes are evaluated for two or more alternative courses of action can a study claim to address the problems that result from limited resources and ever-increasing demands.

Within SCI research, a challenging consideration concerns the type of outcome measure to incorporate into an economic evaluation. The dominant (and often compulsory) practice of outcome measurement for economic evaluation requires the use of generic preference-based health-related quality of life (HRQoL) measures, such as the EQ-5D, SF-6D or Health Utilities Index. ${ }^{1,5}$ The primary purpose of such measures is to provide health state preference scores or utility values for the estimation of quality-adjusted life years (QALYs). The appeal of this approach is that it incorporates value into the outcomes obtained and permits direct comparisons across clinical areas, thereby providing decision makers with a mechanism for resource allocation from a single health-care budget.

A recent systematic review highlighted the absence of supportive evidence regarding the appropriateness of any existing preferencebased HRQoL instrument for SCI populations. ${ }^{5}$ Accordingly, published studies exploring the cost-effectiveness of interventions for SCI-related conditions and complications fall into one of two categories: (i) quantifying outcomes in SCI-related units or (ii) using 
utility measures that lack evidence of empirical validity. Both categories are suboptimal. The former fails to provide decision makers with evidence that allows for direct comparison of competing claims for health-care resources across clinical specialties. Regarding the second category, health state preference scores for SCI populations have been reported in the literature, but, as yet, there is limited evidence from which to infer whether such values are meaningful. ${ }^{6}$

Further outcomes research-conceptual, qualitative and empirical-is necessary to explore the appropriate manner in which to integrate health benefit into economic evaluations for SCI interventions. An important first step is psychometric evaluation of existing utility measures in SCI populations. Areas for longer-term outcomes research will be largely dependent on the performance of current metrics.

\section{CONCLUDING COMMENTS}

There are many reasons why the SCI literature is not resplendent with applied health economic research; the absence of large, pragmatic randomized controlled trials and skepticism toward the measurement of generic health benefit are likely factors. An argument that there are 'bigger fish to fry', whether basic science or best practice considerations, also has merit. However, to compete for scarce health care funding (and, importantly, increasingly scarce research funding), the need to demonstrate the potential for cost-effective and/or cost-containing treatments requires a targeted and focused research agenda.

It is our belief that the paucity of current evidence is an opportunity rather than a hindrance to bring together health economics and spinal cord injury research.

\section{CONFLICT OF INTEREST}

The authors declare no conflict of interest.

\section{ACKNOWLEDGEMENTS}

We would like to thank two anonymous reviewers for their helpful and constructive comments.

DGT Whitehurst ${ }^{1,2,3}$ and N Mittmann ${ }^{4,5,6}$

${ }^{1}$ Faculty of Health Sciences, Simon Fraser University,

Burnaby, British Columbia, Canada;

${ }^{2}$ Centre for Clinical Epidemiology \& Evaluation, Vancouver Coastal

Health Research Institute, Vancouver, British Columbia, Canada;

${ }^{3}$ International Collaboration On Repair Discoveries (ICORD), Vancouver, British Columbia, Canada;

${ }^{4}$ Health Outcomes and PharmacoEconomics (HOPE) Research Centre, Sunnybrook Health Sciences Centre, Toronto, Ontario, Canada;

${ }^{5}$ Department of Pharmacology, University of Toronto, Toronto, Ontario, Canada and

${ }^{6}$ The International Centre for Health Innovation, Richard Ivey School of Business, University of Western Ontario, London, Ontario, Canada E-mail: david_whitehurst@sfu.ca

1 National Institute for Health and Care Excellence. Guide to the Methods of Technology Appraisal 2013. National Institute for Health and Care Excellence: London, UK, 2013.

2 Mittmann N, Seung SJ, Hill MD, Phillips SJ, Hachinski V, Coté R et al. Impact of disability status on ischemic stroke costs in Canada in the first year. Can J Neurol Science 2012; 39: 793-800.

3 Sinnott PL, Cheng A, Wagner TH, Goetz LL, Ottomanelli L. Cost-effectiveness analysis of the spinal cord injury vocational integration program (SCI-VIP). Topics in SCl Rehabil 2011; 16: 80-88.

4 National Cancer Institute of Canada Clinical Trials Group (NCIC CTG); Committee on Economic Analysis http://www.ctg.queensu.ca/committees.html. Accessed 12 February 2013

5 Whitehurst DG, Noonan VK, Dvorak MF, Bryan S. A review of preference-based healthrelated quality of life questionnaires in spinal cord injury research. Spinal Cord 2012; 50: 646-654.

6 Lee BB, King MT, Simpson JM, Haran MJ, Stockler MR, Marial 0 et al. Validity, responsiveness, and minimal important difference for the SF-6D health utility scale in a spinal cord injured population. Value Health 2008; 11: 680-688. 